Supporting Information for:

\title{
PEGylated Nanoparticles obtained Through Emulsion Polymerization as Paclitaxel Carriers
}

\section{Characterization of HEMA-CL $\mathrm{L}_{3}$ macromonomer}

Both the HEMA-CL 3 and the copolymer shell have been characterized via ${ }^{1} \mathrm{H}-\mathrm{NMR}$ and GPC. The ${ }^{1} \mathrm{H}-\mathrm{NMR}$ spectra of both compounds are reported below.

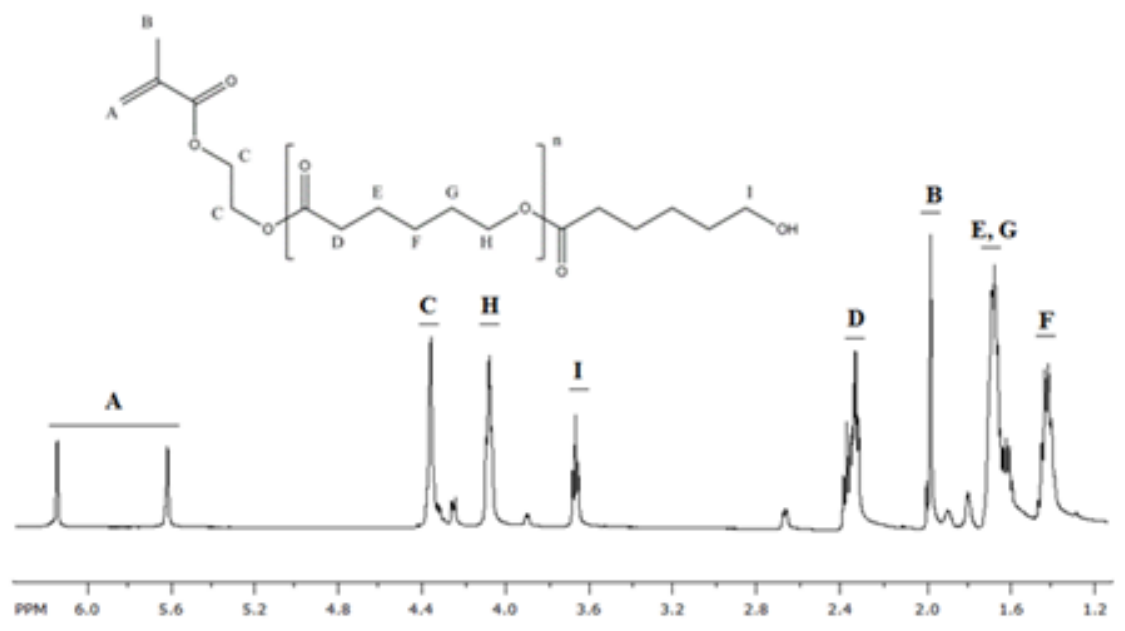

Figure S1 ${ }^{1} \mathrm{H}-\mathrm{NMR}$ spectrum of the HEMA-CL $\mathrm{C}_{3}$ macromonomer

According to the reported spectrum the molecular weight of the macromonomer can be calculated as follows:

$M n=M_{H E M A}+M_{C L}\left[\frac{H \text { (methylene proton signal })}{I(\alpha \text { methylene proton signal })}+1\right]$

EQ S1

The term into brackets represents the average number of CL units that are attached at the HEMA molecule and therefore it is the main parameter that characterized all the produce macromonomer and NPs. It is derived as the ratio between the repeating CL units, $\mathrm{H}$, and the terminal CL unit, I, which is then added to the total count. All the area integrations have been made by setting the area of one of the vinyl hydrogen atoms of the HEMA molecule, A, as the unit. The MW from NMR was calculated es equal to 483 Da. 
The macromonomer average MW and distribution were characterized by size exclusion chromatography (SEC) analysis, using THF as eluent with $0.6 \mathrm{~mL} / \mathrm{min}$ flow rate at a temperature of $35^{\circ} \mathrm{C}$. The instrument (Agilent, 1100 series, Germany) is equipped with differential refractive index (RI), three PLgel columns (Polymer laboratories Ltd., UK; two with pore sizes of MXC type and one oligopore; length of $300 \mathrm{~mm}$ and $7.5 \mathrm{~mm} \mathrm{ID)}$ and a precolumn. Universal calibration was applied, based on polystyrene standards from 580 Da to 3,250,000 Da (Polymer Laboratories). The PDI of the macromonomer was found equal to 1.16

\section{Reproducibility of Nanoparticle Synthesis}

In Table S1 are reported the DLS analysis for different batches of NPs. Each value is the average of three different synthesis

Table S1: DLS analysis of different NP synthesis

\begin{tabular}{ccc}
\hline Sample & Size \pm s.d. & PDI \\
& {$[\mathbf{n m}]$} & {$[-]$} \\
\hline $\mathrm{PCL}_{3}$ blank NPs & $109 \pm 5$ & $0.07 \pm 0.01$ \\
$\mathrm{PCL}_{3} \mathrm{NPs}$ & $113 \pm 7$ & $0.09 \pm 0.01$ \\
\hline
\end{tabular}

It is possible to see from the data reported in Table $\mathrm{S} 1$ that emulsion polymerization is indeed able to guarantee good reproducibility on NP synthesis.

\section{Schematization of the drug loading device}

In the following figure a schematization of the mixing device used for the loading step is presented.

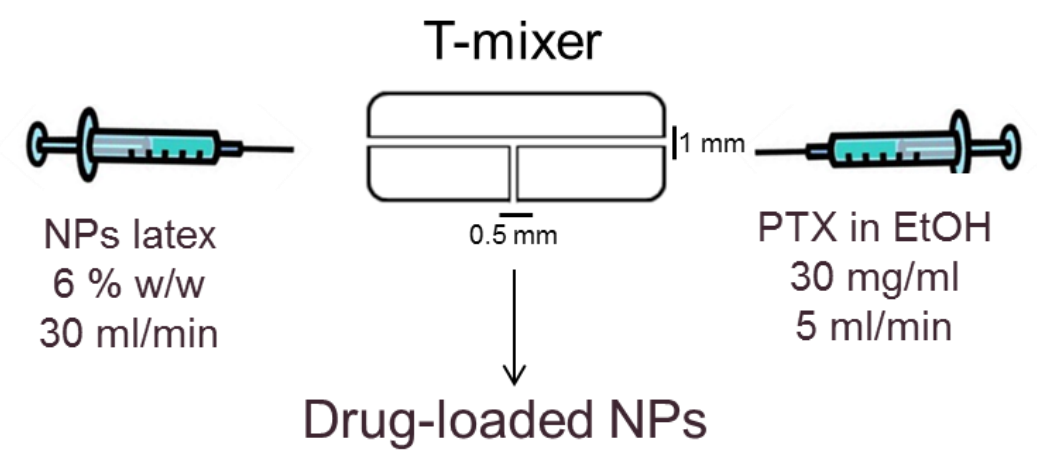

Figure S2: schematic representation of the mixing device and the loading procedure 


\section{Core-Shell Nanoparticles}

In order to try to slow the release of PTX after intravenous injection $\mathrm{PCL}_{3}$ NPs were coated with a PEGylated copolymer shell composed of the HEMA-CL ${ }_{3}$ macromonomer and HEMA-PEG 38 (10\% w/w). . In detail $900 \mathrm{mg}$ of HEMA-CL 3 and $100 \mathrm{mg}$ of HEMA-PEG 38 were dissolved into $3 \mathrm{ml}$ of ethanol and put in a $10 \mathrm{ml}$ glass flask; AIBN (40 mg) was added as a lipophilic radical initiator after the system was purged through nitrogen-vacuum cycles and left under nitrogen atmosphere. The reaction was carried out overnight at $60^{\circ} \mathrm{C}$ in nitrogen atmosphere; afterwards the produced polymer was precipitated in dry cold diethyl ether in order to remove unreacted macromonomers. The final product was than dissolved in ethanol at a concentration of $60 \mathrm{mg} / \mathrm{ml}$.

Characterization of the copolymer was carried out by ${ }^{1} \mathrm{H}-\mathrm{NMR}$ and GPC analysis using the same instruments and parameters used for the HEMA- $\mathrm{CL}_{3}$ macromonomer.

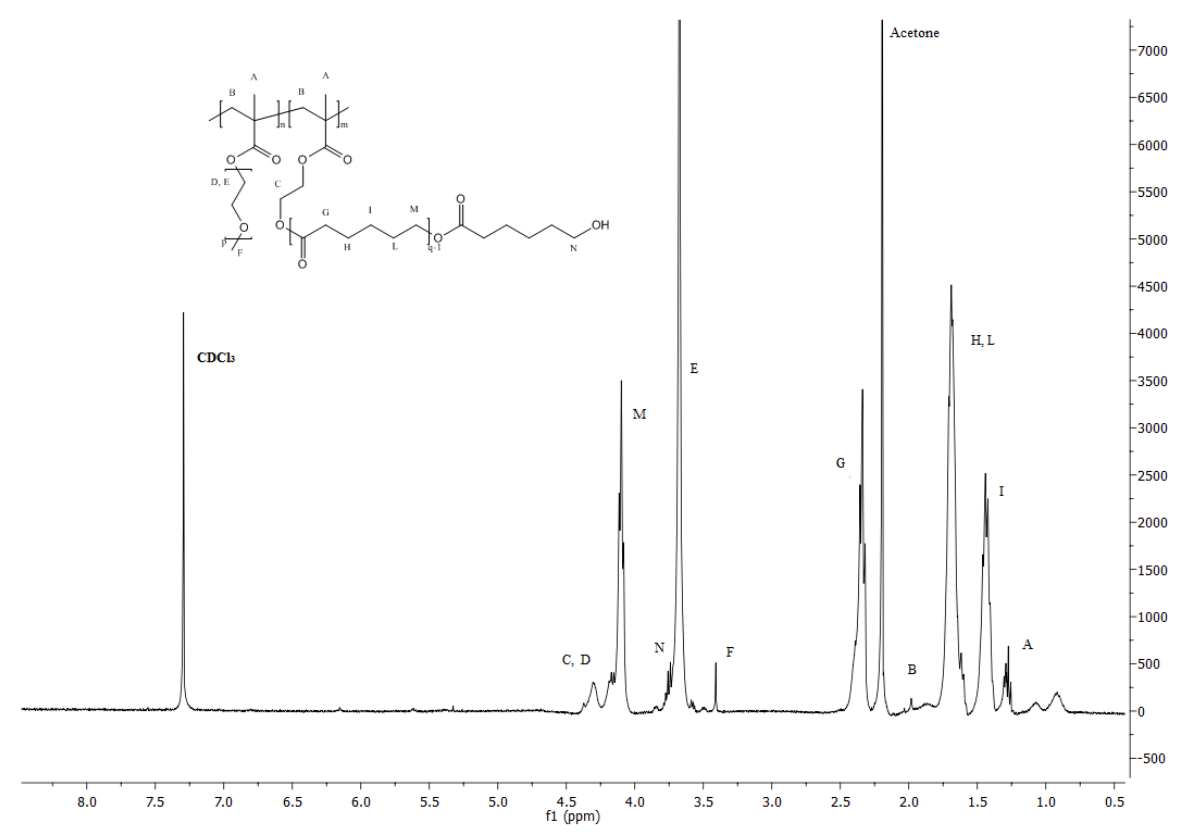

Figure S3 ${ }^{1} \mathrm{H}-\mathrm{NMR}$ spectrum of the copolymer shell together with peak assignement. The molecular weight as determined by GPC is $27381 \mathrm{Da}$ with a PD of 2.31

Core-shell NPs are produced by coating $\mathrm{PCL}_{3}$ NPs with the polymer produced as previously described using a modified nanoprecipitation process on preformed NPs through the same mixing device. In this case the two phases were $\mathrm{PCL}_{3}$ NPs and polymer chains dissolved in ethanol $(60 \mathrm{mg} / \mathrm{ml})$ with a flow rate of $30 \mathrm{ml} / \mathrm{min}$ and $5 \mathrm{ml} / \mathrm{min}$ respectively. Using these conditions it is possible to obtain an increase of the NP diameter 
(from $110 \mathrm{~nm}$ to $122 \mathrm{~nm}$ ) without the formation of neither aggregates nor secondary NP distribution, as confirmed by DLS analysis.

Core-Shell NPs have been employed in the same in vivo model adopted for the evaluation of therapeutic efficacy and PTX biodistribution used for $\mathrm{PCL}_{3}$ NPs. Results are reported in the figure below.
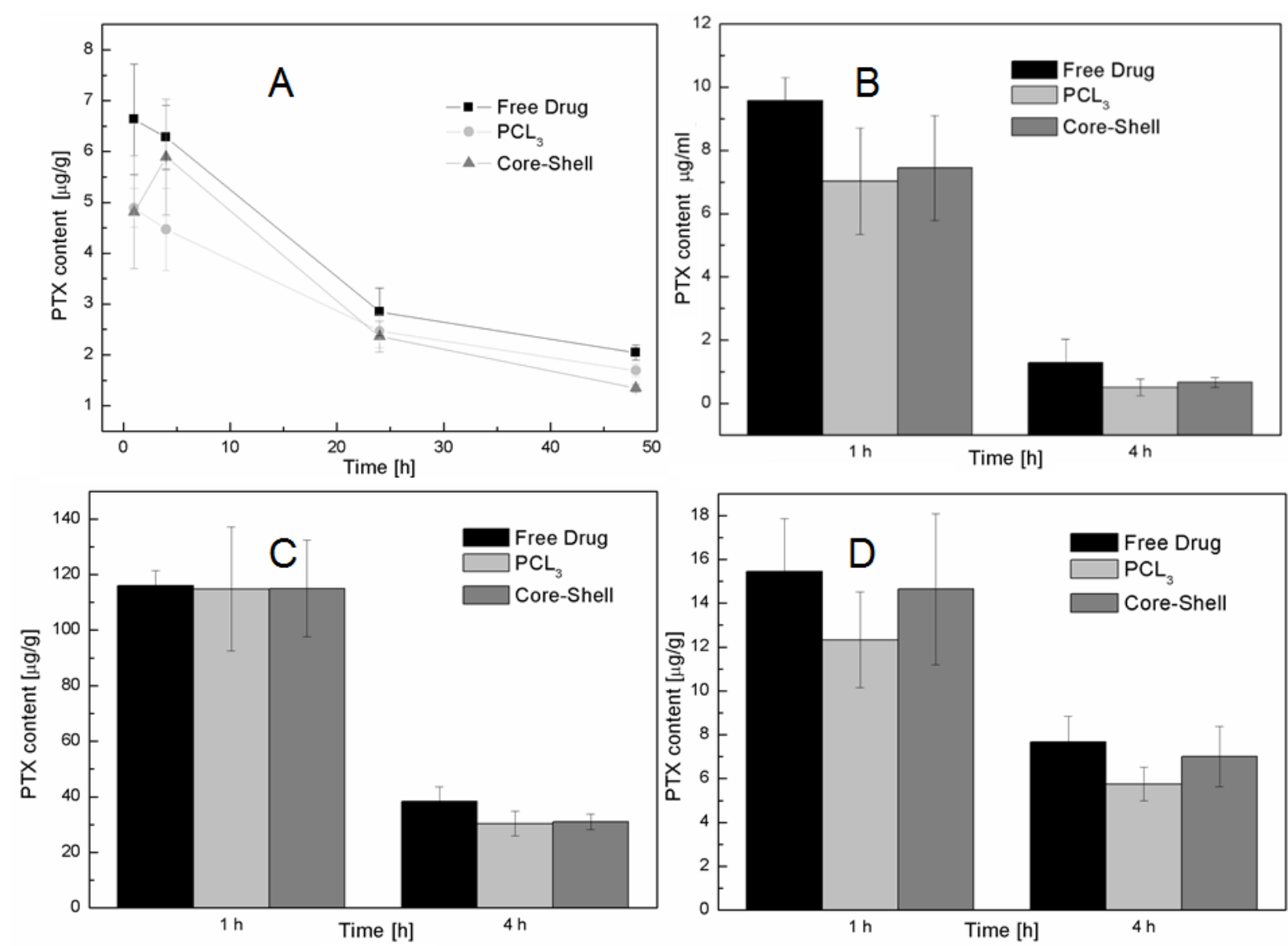

Figure S4 Comparison between PTX recovered in the tumor (panel A), plasma (panel B) liver (panel C) and spleen (panel D) for the different formulations.

From the data reported in Figure S4 it is clearly possible to see that the addition of a polymer shell does not lead to a significant change in PTX distribution if compared to the standard formulation or $\mathrm{PCL}_{3} \mathrm{NPs}_{\text {. This }}$ can be explained with literature evidence in which it is postulated that amphiphilic polymer adsorbed on the NPs can be displaced form the NP surface by plasma proteins possessing a strong affinity for components on the NPs themselves. The similar PTX level in the tumor lead to a comparable therapeutic activity of the formulation compared to $\mathrm{PCL}_{3}$ NPs and the free drug (data not shown). Therefore it is possible to conclude 
that the addition of a polymer shell represent a further complication in the design of PTX-loaded NPs which does not lead to an improvement in their activity. 